EPJ Web of Conferences 41, 05019 (2013)

DOI: $10.1051 /$ epjconf/20134105019

(C) Owned by the authors, published by EDP Sciences, 2013

\title{
Ultrafast IR pump-probe and 2D-IR photon echo spectroscopy of adenosine-thymidine base pairs
}

\author{
Christian Greve ${ }^{1}$, Nicholas K. Preketes ${ }^{2}$, Rene Costard ${ }^{1}$, Benjamin Koeppe ${ }^{1}$, Henk Fidder ${ }^{1}$, \\ Erik T. J. Nibbering ${ }^{1}{ }^{1 *}$, Friedrich Temps ${ }^{3}$, Shaul Mukamel $^{2}$, and Thomas Elsaesser ${ }^{1}$ \\ ${ }^{1}$ Max-Born-Institut für Nichtlineare Optik und Kurzzeitspektroskopie, Max-Born-Str. 2 A, D-12489 \\ Berlin, Germany; E-mail: nibberin@mbi-berlin.de \\ ${ }^{2}$ Department of Chemistry, University of California, Irvine, California 92697-2025, USA \\ ${ }^{3}$ Institut für Physikalische Chemie, Christian-Albrechts-Universität zu Kiel, Olshausenstr. 40, D- \\ 24098 Kiel, Germany
}

\begin{abstract}
We characterize diagonal and off-diagonal anharmonicities of $\mathrm{N}-\mathrm{H}$ stretching vibrations in adenosine and thymidine monomers and in $\mathrm{A} \bullet \mathrm{T}$-base pairs in chloroform solution, showing the important role of coupling between vibrationally excited N-H stretching states.
\end{abstract}

Hydrogen bonding between nucleic acid bases, the building blocks of the genetic code in DNA or RNA, has been studied by numerous experimental and theoretical methods. Typically one follows either a top-down approach, where a DNA (or RNA) double helix formed by a specific base pair sequence is studied [1,2], or a bottom-up approach, where only a limited number of building blocks, i.e. nucleic acid bases, are included in the study [3]. We have followed the latter approach using chemically modified guanine $(\mathrm{G})$ and cytosine $(\mathrm{C})$ nucleic bases to warrant solubility in weakly polar chloroform and a preferred Watson-Crick (WC) hydrogen bonding geometry. We have assigned the $\mathrm{N}-\mathrm{H}$ stretching bands of $\mathrm{G} \bullet \mathrm{C}$ base pairs observed in the linear FT-IR spectrum using a local mode picture, and elucidated the diagonal and off-diagonal cross peaks measured with femtosecond 2D-IR photon echo spectroscopy [4]. Using the same approach we present here new results on adenosinethymidine $(\mathrm{A} \bullet \mathrm{T})$ base pairs, chemically modified in the same way as $\mathrm{G}$ and $\mathrm{C}$, dissolved in chloroform. To obtain a general understanding of the linear and nonlinear spectroscopy of the N-H stretching manifolds of different complexation geometries of $\mathrm{A} \bullet \mathrm{T}$, we first explore the nonlinear response of $\mathrm{N}-\mathrm{H}$ stretching modes of $\mathrm{A}$ and $\mathrm{T}$ monomers. For a mixture of $\mathrm{A}$ and $\mathrm{T}$ in chloroform solution, $\mathrm{A}$ and $\mathrm{T}$ monomers and four different complexation geometries are present. The highly congested 2D-IR spectra of such mixtures are disentangled with the help of the separately measured monomer 2D-IR spectra. The diagonal and off-diagonal anharmonic couplings between the N-H stretching modes have been calculated at the HF/6-311++G** level.

Fig. 1 shows the linear and 2D-IR spectra recorded for A monomers and A $\bullet T$ base pairs. In the case of A monomer, the linear spectra show the symmetric and asymmetric $\mathrm{NH}_{2}$-stretching modes at $3413 \mathrm{~cm}^{-1}$ and at $3525 \mathrm{~cm}^{-1}$, indicating a coupling strength of $J=-56 \mathrm{~cm}^{-1}$ between the $\mid 1,0>$ and $\mid 0,1>$ local N-H stretching modes. The diagonal and off-diagonal peaks 2D-IR spectra can be fully resolved for A monomer, therefore, all transitions can be determined for the single and double quantum excitation manifolds [5]. The energy levels of the one and two-exciton manifolds were calculated at the HF/6-311++G** level by a sixth-order expansion of the Hamiltonian in the two N-

This is an Open Access article distributed under the terms of the Creative Commons Attribution License 2.0, which permits unrestricted use, distribution, and reproduction in any medium, provided the original work is properly cited. 
$\mathrm{H}$ stretching modes. The resulting comparison between experiment and theory enables to determine in detail the diagonal anharmonicities and anharmonic couplings of the N-H stretching modes, either using the normal or the local mode representations. It turns out that for the single-quantum excitation manifold the normal mode picture with symmetric and asymmetric $\mathrm{N}-\mathrm{H}$ stretching modes is fully appropriate. For the two-quantum excitation manifold, on the other hand, the interplay between intermode coupling and large diagonal anharmonic frequency shifts leads to a situation in which mixing is much weaker. Such a finding is fully in line with reported results on overtone spectroscopy of coupled hydrogen stretching oscillators [6].

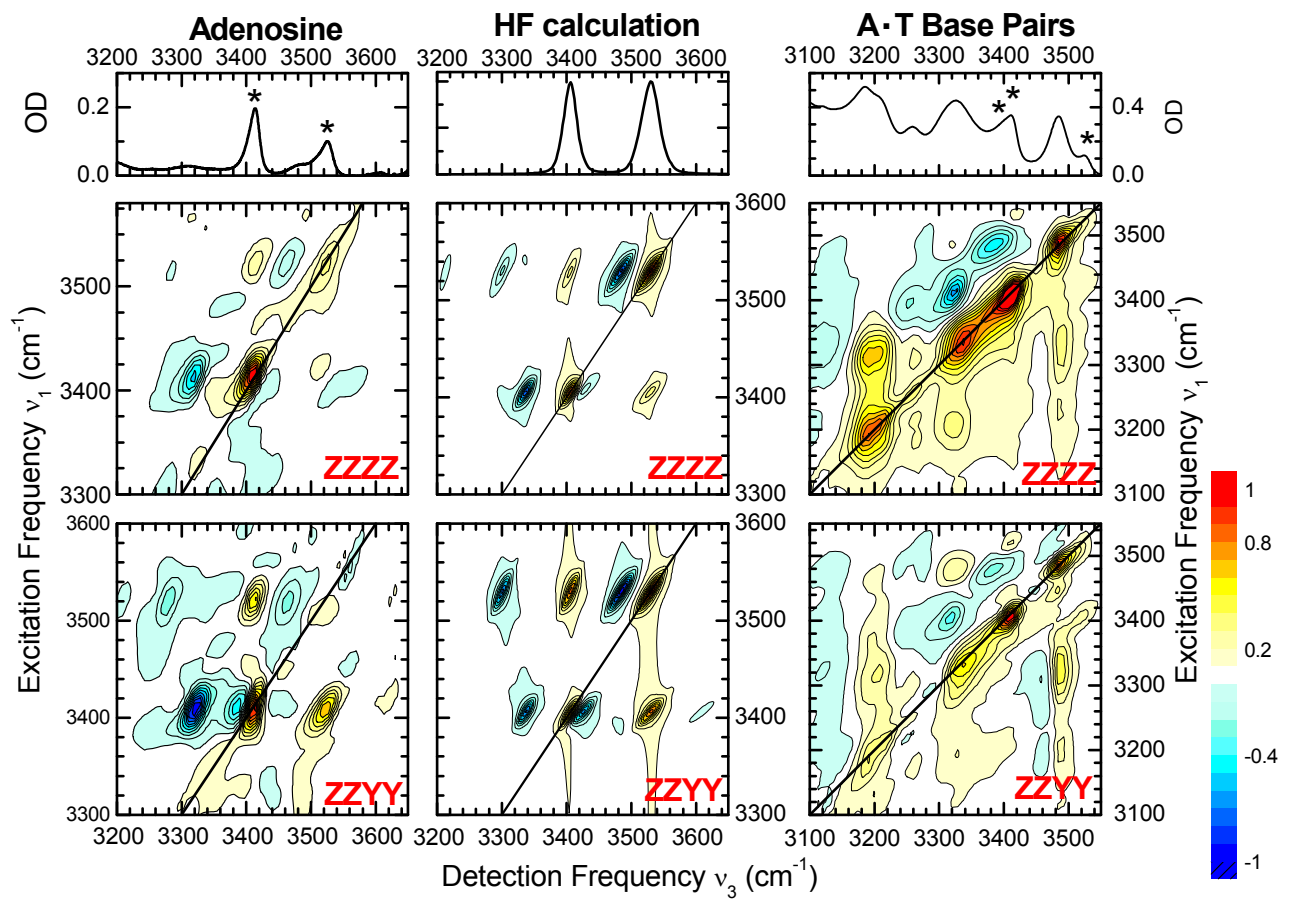

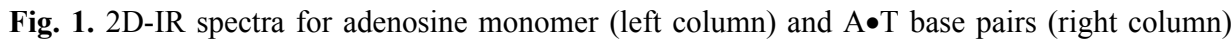
recorded at a population waiting time of $200 \mathrm{fs}$, using the (ZZZZ) and (ZZYY) polarisation conditions (second and third row, respectively). Calculated 2D-IR spectra for A monomer are given in the second column. The linear FT-IR spectra are depicted in the top panels. Asterisks denote $\mathrm{N}-\mathrm{H}$ stretching bands for the $\mathrm{A}$ and $\mathrm{T}$ monomers.

When both $\mathrm{A}$ and $\mathrm{T}$ are present in chloroform solution, the FT-IR spectra show bands related to $\mathrm{A} \bullet \mathrm{T}$ base pairs, as well as A and $\mathrm{T}$ monomer bands. Four different base pairing geometries must be considered in the analysis of the data: WC, reverse Watson-Crick (rWC), Hoogsteen $(\mathrm{H})$ and reverse Hoogsteen $(\mathrm{rH})$. This sharply contrasts with our previous study of $\mathrm{G} \bullet \mathrm{C}$ base pairs where the WatsonCrick (WC) geometry dominates [4]. From NMR measurements on A $\bullet T$ low temperature Freon solutions, as well as ab-initio self-consistent reaction field calculations, we conclude that all four complexes have similar energetic stabilities in chloroform solution, with WC and rWC slightly more stable than $\mathrm{H}$ and $\mathrm{rH}$ complexes. Concentration and temperature-dependent FT-IR measurements of A $\bullet T$ solutions show that the N-H stretching manifold in the $3100-3600 \mathrm{~cm}^{-1}$ range has at least three main bands at 3188,3324 and $3482 \mathrm{~cm}^{-1}$. A closer inspection reveals a shoulder at 3211 and a smaller peak at $3262 \mathrm{~cm}^{-1}$ as well as a distinct spectral tail extending below the $\mathrm{C}-\mathrm{H}$ stretching manifold down to $2600 \mathrm{~cm}^{-1}$. Ab initio calculations of the frequencies and transition dipoles of the N$\mathrm{H}$ stretching vibrations shows that the local mode description prevails in all possible $\mathrm{A} \bullet \mathrm{T}$ base pairs. Assignment of these different contributions in the linear FT-IR spectra to particular A $\bullet \mathrm{T}$ base pairs geometries, however, remains impossible. Only with femtosecond two-colour IR-pump-IR-probe 
and 2D-IR spectroscopy we can unequivocally show that the multitude of N-H stretching transitions all originate from the same $\mathrm{A} \bullet \mathrm{T}$ base pairs, i.e. the mode description clearly goes beyond the simple model of having only three transitions due to three N-H stretching modes per base pair.

2D-IR photon echo experiments on the A $\bullet \mathrm{T}$ solutions using the $\left(\boldsymbol{k}_{1}, \boldsymbol{k}_{2}, \boldsymbol{k}_{3}, \boldsymbol{k}_{L O}\right)$ polarization configurations (ZZZZ) and (ZZYY) reveal that N-H stretching bands at 3188,321 and $3324 \mathrm{~cm}^{-1}$ associated with hydrogen-bonded $\mathrm{N}-\mathrm{H}$ groups of $\mathrm{A}$ and $\mathrm{T}$ have cross peaks with the narrow N-H stretching band located at $3482 \mathrm{~cm}^{-1}$. Comparing this with the known band splitting for the symmetric and asymmetric N-H stretching modes of A monomer, it follows that the narrow band at $3482 \mathrm{~cm}^{-1}$ is due to the free $\mathrm{N}-\mathrm{H}$ group of $\mathrm{A} \bullet \mathrm{T}$. Hydrogen bonding of the $\mathrm{NH}_{2}$-group of $\mathrm{A}$ to the carbonyl moiety of $\mathrm{T}$ leads to a frequency downshift of the hydrogen bonded $\mathrm{N}-\mathrm{H}$ stretching mode, thereby effectively decoupling the two local $\mathrm{NH}$-modes of the $\mathrm{NH}_{2}$-group.

Comparing frequency-resolved pump-probe measurements on $\mathrm{A}$ and $\mathrm{T}$ monomers with those on $\mathrm{A} \bullet \mathrm{T}$, a significant shortening of the $\mathrm{N}-\mathrm{H}$ stretching lifetimes is found. Whereas both the symmetric and asymmetric N-H stretching modes of A monomer have a $T_{l}=6 \mathrm{ps}$, the N-H stretching vibration of $\mathrm{T}$ monomer has a value of $T_{1}=0.9 \mathrm{ps}$ (the latter is similar to the value reported for uracil monomer [3]). The unbound (free) $\mathrm{N}-\mathrm{H}$ stretching mode of $\mathrm{A} \bullet \mathrm{T}$ has $T_{l}=1.8 \mathrm{ps}$. In contrast, the lifetimes of the hydrogen-bonded N-H stretching modes of A•T range from $0.2-0.4$ ps.
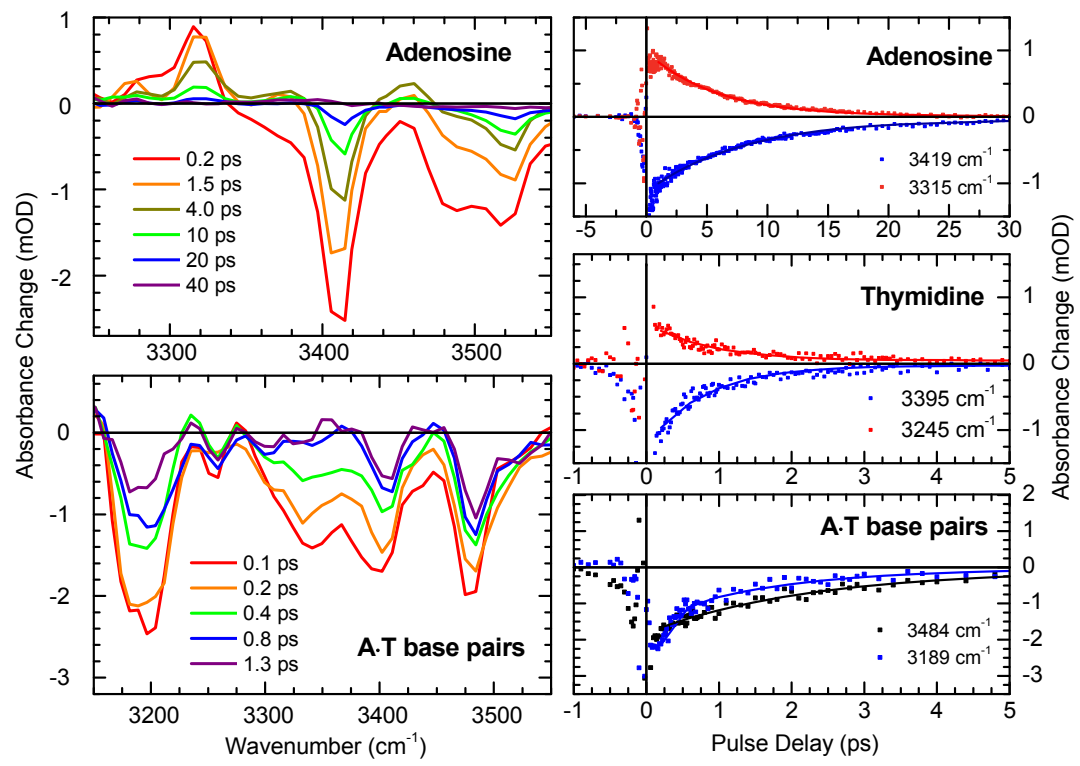

Fig. 2. Pump-probe kinetics measured on $A$ and $T$ monomers, as well as on $A \bullet T$ base pairs. Transient spectra are shown in the left column, kinetic traces of excited state absorption and ground state bleach signals are shown in the right column.

\section{References}

1. Ł. Szyc, M. Yang, E. T. J. Nibbering, T. Elsaesser, Angew. Chem. Int. Ed. 49, 3598 (2010).

2. M. Yang, Ł. Szyc, T. Elsaesser, J. Phys. Chem. A 115, 1262 (2011).

3. S. Woutersen, G. Cristalli, J. Chem. Phys. 121, 5381 (2004).

4. M. Yang, Ł. Szyc, K. Röttger, H. Fidder, E. T. J. Nibbering, T. Elsaesser, F. Temps, J. Phys. Chem. A 115, 5484 (2011).

5. C. Greve, N. K. Preketes, R. Costard, B. Koeppe, H. Fidder, E. T. J. Nibbering, F. Temps, S. Mukamel, T. Elsaesser, J. Phys. Chem. A 115, in press (2011); DOI: 10.1021/jp303864m .

6. M. S Child and L. Halonen, Adv. Chem. Phys. 57, 1 (1984). 